\title{
Robust Stability Criteria of Roesser-Type Discrete-Time Two-Dimensional Systems with Parameter Uncertainties
}

\author{
Yan Zhao, ${ }^{1}$ Tieyan Zhang, ${ }^{1}$ Dan Zhao, ${ }^{1}$ Fucai You, ${ }^{1}$ and Miao $\mathrm{Li}^{2}$ \\ ${ }^{1}$ School of Renewable Energy, Shenyang Institute of Engineering, Shenyang, Liaoning 110136, China \\ ${ }^{2}$ School of Engineering and Information Technology, Murdoch University, Perth, WA 6150, Australia \\ Correspondence should be addressed to Yan Zhao; zhaoyan_80@163.com
}

Received 9 January 2014; Accepted 2 February 2014; Published 16 March 2014

Academic Editor: Ming Liu

Copyright (c) 2014 Yan Zhao et al. This is an open access article distributed under the Creative Commons Attribution License, which permits unrestricted use, distribution, and reproduction in any medium, provided the original work is properly cited.

\begin{abstract}
This paper is concerned with robust stability analysis of uncertain Roesser-type discrete-time two-dimensional (2D) systems. In particular, the underlying parameter uncertainties of system parameter matrices are assumed to belong to a convex bounded uncertain domain, which usually is named as the so-called polytopic uncertainty and appears typically in most practical systems. Robust stability criteria are proposed for verifying the robust asymptotical stability of the related uncertain Roesser-type discretetime 2D systems in terms of linear matrix inequalities. Indeed, a parameter-dependent Lyapunov function is applied in the proof of our main result and thus the obtained robust stability criteria are less conservative than the existing ones. Finally, the effectiveness and applicability of the proposed approach are demonstrated by means of some numerical experiments.
\end{abstract}

\section{Introduction}

During the past several decades, the well-known Lyapunov stability theory has become an efficient tool for dealing with the problem of stability analysis of many kinds of uncertain systems [1-6]. However, those earlier results on stability analysis of uncertain systems are developed by using the socalled common quadratic Lyapunov function (CQLF) [7]. Actually, the CQLF applies a single Lyapunov matrix for all the submodels and therefore the obtained stability criteria are rather conservative. With the purpose of further releasing the conservatism of the stability criteria, the affine parameterdependent Lyapunov function (APDLF) has been proposed in [8], where the fixed quadratic Lyapunov function is replaced by a Lyapunov function with affine dependence on the underlying uncertain parameters. Because of the construction of such parameter-dependent Lyapunov functions, the conservatism could be released a lot as a tradeoff.

On the other hand, the famous 2D systems model could represent a wide range of practical plants, for example, water stream heating, thermal processes, biomedical imaging, gas absorption, river pollution modeling, data processing and transmission, process of gas filtration, grid based wireless sensor networks, and so forth, $[9,10]$. As a result, a considerable interest in stability analysis of 2D systems has emerged during the past two decades [11-15]. Recently, the 2D system theory has also been applied to address the problem of stability analysis $2 \mathrm{D}$ state-space digital filters with saturation arithmetic in [16-30]. However, it is worth noting that most of the aforementioned results are feasible for linear 2D systems without uncertainties. As is well known, most of the practical $2 \mathrm{D}$ dynamical systems in the realistic world are subject to parameter uncertainties and the above results would fail to work when some uncertain parameters occur in the practical settings.

In particular, it is worth noting that the Roesser-type discrete-time 2D system's information is propagated along two independent directions and this fact makes the problem of stability analysis more complicated. Due to the complexity of mathematical analysis of Roesser-type discrete-time 2D systems with parameter uncertainties, there has been little literature which focuses on robust stability analysis of uncertain Roesser-type discrete-time 2D systems so far. Thus, this problem needs to be further investigated and this fact motivates us to carry out this task in this paper.

Based on the above analysis, the problem of robust stability analysis of Roesser-type discrete-time 2D systems with parameter uncertainties will be addressed via the Lyapunov stability theory. The parameter uncertainties of 2D system's 
parameter matrices are assumed to belong to a convex bounded uncertain domain, which usually is named as the so-called polytopic uncertainty and appears typically in most modeling processes of uncertainties. An efficient parameterdependent Lyapunov function is applied in the derivation of our main result and thus the obtained robust stability criteria are less conservative than the existing ones. Moreover, robust stability criteria are given to verify the robust asymptotical stability of the uncertain Roesser-type discrete-time 2D systems in terms of linear matrix inequalities. Finally, the effectiveness and applicability of the proposed approach are demonstrated by means of numerical examples.

The rest of this paper is organized as follows: following the introduction, some preliminaries are provided in Section 2. In Section 3, LMI-based robust stability criteria are proposed for verifying the robust asymptotical stability of the uncertain Roesser-type discrete-time 2D systems. A numerical example is given to demonstrate the effectiveness of the given approach in Section 4. Finally, some conclusions are also given in Section 5.

The following notations are applied for simplicity. A star * in a symmetric matrix denotes the transposed element in the symmetric position; the symbol $I$ represents the identity matrix with appropriate dimension; $X>0$ (or $X \geq 0$ ) means the matrix $X$ is symmetric and positive definite (or symmetric and positive semidefinite); $X^{T}$ denotes the transpose of $X$.

\section{Preliminaries}

Consider a class of uncertain discrete-time 2D systems which is described by the Roesser-type model

$$
\mathbf{x}^{+}(k, l)=A(\alpha) \mathbf{x}(k, l),
$$

with

$$
\mathbf{x}(k, l)=\left[\begin{array}{l}
\mathbf{x}^{h}(k, l) \\
\mathbf{x}^{v}(k, l)
\end{array}\right], \quad \mathbf{x}^{+}(k, l)=\left[\begin{array}{l}
\mathbf{x}^{h}(k+1, l) \\
\mathbf{x}^{v}(k, l+1)
\end{array}\right],
$$

where $k$ and $l$ are two integers in $\mathbb{Z}^{+} . \mathbf{x}^{h}(\cdot, \cdot)$ is the horizontal state in $\mathbf{R}^{n_{1}}$ and $\mathbf{x}^{v}(\cdot, \cdot)$ is the vertical state in $\mathbf{R}^{n_{2}}$, where $n_{1}$ and $n_{2}$ are dimensions of the horizontal state vector and the vertical state vector, respectively. The system coefficient matrix $A(\alpha)$ is not precisely known but belongs to a convex bounded uncertain domain:

$$
A(\alpha)=\left[\begin{array}{lll}
A^{11}(\alpha) & A^{12}(\alpha) \\
A^{21} & (\alpha) & A^{22}(\alpha)
\end{array}\right]
$$

with $A^{11}(\alpha) \in \mathbf{R}^{n_{1} \times n_{1}}, A^{12}(\alpha) \in \mathbf{R}^{n_{1} \times n_{2}}, A^{21}(\alpha) \in \mathbf{R}^{n_{2} \times n_{1}}$, and $A^{22}(\alpha) \in \mathbf{R}^{n_{2} \times n_{2}}$, respectively. Specially, these matrices $A^{11}(\alpha), A^{12}(\alpha), A^{21}(\alpha)$, and $A^{22}(\alpha)$ belong to a convex bounded (polytope type) uncertain domain $\mathscr{P}$ given as follows:

$$
\begin{gathered}
\mathscr{P}:=\left\{\left(A^{11}, A^{12}, A^{21}, A^{22}\right)(\alpha):\left(A^{11}, A^{12}, A^{21}, A^{22}\right)(\alpha)\right. \\
\left.=\sum_{i=1}^{r} \alpha_{i}\left(A_{i}^{11}, A_{i}^{12}, A_{i}^{21}, A_{i}^{22}\right) ; \alpha \in \Delta_{r}\right\},
\end{gathered}
$$

where $\Delta_{r}$ is the so-called unit simplex given by

$$
\Delta_{r}=\left\{\alpha \in \mathbf{R}^{r}: \sum_{i=1}^{r} \alpha_{i}=1, \alpha_{i} \geq 0 ; i=1, \ldots, r\right\} .
$$

Moreover, the boundary conditions along two independent directions are defined as $\mathbf{x}^{h}(0, l)=f(l)$ and $\mathbf{x}^{v}(k, 0)=g(k)$, where $f(l)$ and $g(k)$ are boundary conditions along the horizontal direction and vertical direction, respectively.

Finally, let us end this section by giving a definition and a lemma which will play an important role in the following proof.

Denote $X_{N}=\sup \{\|\mathbf{x}(k, l)\|: N=k+l\}$, and then we give the definition of robust asymptotical stability for uncertain Roesser-type discrete-time 2D system (1).

Definition 1. The uncertain Roesser-type discretetime 2D system (1) is robust asymptotically stable if $\lim _{k \rightarrow \infty, l \rightarrow \infty} X_{N}=0$ with the initial and boundary conditions $\mathbf{x}^{h}(0, l)=f(l)$ and $\mathbf{x}^{v}(k, 0)=g(k)$.

Lemma 2 (see [7]). Given matrices $Q=Q^{T}$ and $R=R^{T}$ with appropriate dimensions, the inequality $\left(\begin{array}{cc}Q & S \\ S^{T} & R\end{array}\right)>0$ is equivalent to $R>0, Q-S R^{-1} S^{T}>0$.

\section{Main Results}

In this section, by using the Lyapunov stability theory, sufficient robust stability criteria for ensuring the robust asymptotical stability of the underlying uncertain Roesser-type discrete-time 2D system (1) will be proposed in terms of linear matrix inequalities. Indeed, less conservative robust stability conditions are given by means of a parameter-dependent Lyapunov function and a slack method for exploiting the algebraic properties of the uncertain Roesser-type discrete-time 2D system (1).

Theorem 3. The uncertain Roesser-type discrete-time $2 D$ system (1) is robust asymptotically stable if there exist appropriately dimensional matrices $P_{i j}, i=1,2, \ldots, r ; i \leq j \leq r$, with

$$
\begin{gathered}
P_{i j}=\left[\begin{array}{cc}
P_{i j}^{1} & * \\
P_{i j}^{3} & P_{i j}^{2}
\end{array}\right], \quad P_{i j}^{1} \in \mathbf{R}^{n_{1} \times n_{1}}, \\
P_{i j}^{2} \in \mathbf{R}^{n_{2} \times n_{2}}, \quad P_{i j}^{3} \in \mathbf{R}^{n_{2} \times n_{1}},
\end{gathered}
$$

such that the following LMIs hold:

$$
\begin{gathered}
{\left[\begin{array}{cc}
-P_{i i} & * \\
P_{i i} A_{i} & -P_{i i}
\end{array}\right]<0, \quad i=1,2, \ldots, r ;} \\
{\left[\begin{array}{cc}
-P_{i i} & * \\
P_{i i} A_{j} & -P_{i i}
\end{array}\right]+\left[\begin{array}{cc}
-P_{i j} & * \\
P_{i j} A_{i} & -P_{i j}
\end{array}\right]<0,} \\
i=1,2, \ldots, r-1, \quad i<j ;
\end{gathered}
$$




$$
\begin{gathered}
{\left[\begin{array}{cc}
-P_{j j} & * \\
P_{j j} A_{i} & -P_{j j}
\end{array}\right]+\left[\begin{array}{cc}
-P_{i j} & * \\
P_{i j} A_{j} & -P_{i j}
\end{array}\right]<0,} \\
i=1,2, \ldots, r-1, \quad i<j ; \\
{\left[\begin{array}{cc}
-P_{i j} & * \\
P_{i j} A_{l} & -P_{i j}
\end{array}\right]+\left[\begin{array}{cc}
-P_{i l} & * \\
P_{i l} A_{j} & -P_{i l}
\end{array}\right]+\left[\begin{array}{cc}
-P_{j l} & * \\
P_{j l} A_{i} & -P_{j l}
\end{array}\right]<0,} \\
i=1,2, \ldots, r-2, \quad i<j<l \leq r .
\end{gathered}
$$

Proof. Consider the following parameter-dependent Lyapunov function which is suitable for the uncertain Roessertype discrete-time 2D system (1):

$$
V(\mathbf{x}(k, l))=\mathbf{x}^{T}(k, l) P_{\alpha \alpha} \mathbf{x}(k, l),
$$

where the matrix $P_{\alpha \alpha}$ is a positive definite matrix and with the

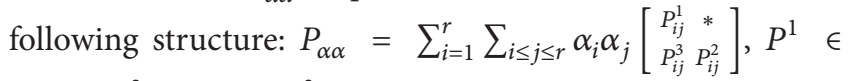
$\mathbf{R}^{n_{1} \times n_{1}}, P^{2} \in \mathbf{R}^{n_{2} \times n_{2}}, P^{3} \in \mathbf{R}^{n_{2} \times n_{1}}$.

Then, the variation of the parameter-dependent Lyapunov function $V(\mathbf{x}(k, l))$ could be described as

$$
\Delta V(\mathbf{x}(k, l))=\mathbf{x}^{T}(k, l)\left(A(\alpha)^{T} P_{\alpha \alpha} A(\alpha)-P_{\alpha \alpha}\right) \mathbf{x}(k, l) .
$$

By applying the Lyapunov stability theory, the uncertain Roesser-type discrete-time 2D system (1) is robust asymptotically stable if the following inequality holds:

$$
A(\alpha)^{T} P_{\alpha \alpha} A(\alpha)-P_{\alpha \alpha}<0
$$

Applying Lemma 2 to (10), it can be concluded that inequality (10) is equivalent to the following inequality:

$$
\Phi=\left[\begin{array}{cc}
-P_{\alpha \alpha} & * \\
P_{\alpha \alpha} A(\alpha) & -P_{\alpha \alpha}
\end{array}\right]<0 .
$$

On the other hand, reordering the expression of $\Psi$, one can obtain

$$
\begin{aligned}
\Phi= & {\left[\begin{array}{cc}
-P_{\alpha \alpha} & * \\
P_{\alpha \alpha} A(\alpha) & -P_{\alpha \alpha}
\end{array}\right] } \\
= & \sum_{i=1}^{r} \alpha_{i}^{3} \Phi_{i i i}+\sum_{i=1}^{r-1} \sum_{j>i} \alpha_{i}^{2} \alpha_{j} \Phi_{i i j} \\
& +\sum_{i=1}^{r-1} \sum_{j>i} \alpha_{i}^{2} \Upsilon_{j} \Gamma_{i j j}+\sum_{i=1}^{r-2} \sum_{j>i} \sum_{l>j} \alpha_{i} \alpha_{j} \alpha_{l} \Phi_{i j l},
\end{aligned}
$$

where we have

$$
\begin{gathered}
\Phi_{i i i}=\left[\begin{array}{cc}
-P_{i i} & * \\
P_{i i} A_{i} & -P_{i i}
\end{array}\right], \\
\Phi_{i i j}=\left[\begin{array}{cc}
-P_{i i} & * \\
P_{i i} A_{j} & -P_{i i}
\end{array}\right]+\left[\begin{array}{cc}
-P_{i j} & * \\
P_{i j} A_{i} & -P_{i j}
\end{array}\right],
\end{gathered}
$$

$$
\begin{gathered}
\Phi_{i j j}=\left[\begin{array}{cc}
-P_{j j} & * \\
P_{j j} A_{i} & -P_{j j}
\end{array}\right]+\left[\begin{array}{cc}
-P_{i j} & * \\
P_{i j} A_{j} & -P_{i j}
\end{array}\right], \\
\Phi_{i j l}=\left[\begin{array}{cc}
-P_{i j} & * \\
P_{i j} A_{l} & -P_{i j}
\end{array}\right]+\left[\begin{array}{cc}
-P_{i l} & * \\
P_{i l} A_{j} & -P_{i l}
\end{array}\right] \\
+\left[\begin{array}{cc}
-P_{j l} & * \\
P_{j l} A_{i} & -P_{j l}
\end{array}\right] .
\end{gathered}
$$

From (10)-(12), if the LMI-based stability conditions (7) hold, inequality (10) evidently holds, which guarantee the robust asymptotical stability for the uncertain Roesser-type discretetime 2D system (1).

This completes the proof.

Remark 4. From (1) and (4), the parameter uncertainties of $2 \mathrm{D}$ system parameter matrices are assumed to belong to a convex bounded uncertain domain. Then, LMI-based robust stability criteria are given for ensuring the robust asymptotical stability of the underlying uncertain Roesser-type discrete-time $2 \mathrm{D}$ systems in Theorem 3 . Indeed, the parameterdependent Lyapunov function $V(\mathbf{x}(k, l))=\mathbf{x}^{T}(k, l) P_{\alpha \alpha} \mathbf{x}(k, l)$ is applied in the derivation of our main result and thus the obtained robust stability criteria are less conservative than before. Furthermore, the effectiveness and applicability of the proposed results will be demonstrated by means of numerical experiments in the following section.

\section{Numerical Examples}

Consider the uncertain Roesser-type discrete-time twodimensional systems described as follows:

$$
\left[\begin{array}{l}
x^{h}(k+1, l) \\
x^{v}(k, l+1)
\end{array}\right]=\sum_{i=1}^{2} \alpha_{i}\left(A_{i}\left[\begin{array}{l}
x^{h}(k, l) \\
x^{v}(k, l)
\end{array}\right]\right)
$$

where $A_{1}=\left[\begin{array}{cc}1+a_{1} T_{1} & \left(a_{1} a_{2}+a_{0}\right) T_{1} \\ T_{2} & 1+a_{2} T_{2}\end{array}\right]$ and $A_{2}=\left[\begin{array}{cc}1+a_{1} T_{1} & a_{1} a_{2} T_{1} \\ T_{2} & 1+a_{2} T_{2}\end{array}\right]$. And the following parameter values about $a_{0}, a_{1}, a_{2}, T_{1}$, and $T_{2}$ are given: $a_{0}=-2, a_{1}=-3, T_{1}=0.1$, and $T_{2}=0.2$. Furthermore, the initial and boundary conditions of the above uncertain Roesser-type discrete-time two-dimensional systems are set as $x^{h}(0, l)=6 \cos (l)$ for $l<30$ and $x^{v}(k, 0)=4 \sin (k)$ for $k<$ 30 and $x^{h}(0, l)=0$ for $l \geq 30$ and $x^{v}(k, 0)=0$ for $k \geq 30$.

Let $a_{2}=-0.6$; the stability criteria given in Theorem 3 are feasible by solving LMIs (7), which guarantee the robust asymptotical stability for the underlying uncertain Roessertype discrete-time $2 \mathrm{D}$ systems. On the other hand, Figures 1 and 2 show the state trajectory of the system state variables $x^{h}(k, l)$ and $x^{v}(k, l)$ with $\alpha_{1}=0.4$ and $\alpha_{2}=0.6$, respectively. From Figures 1 and 2, it is easy to see that the state trajectories of $x^{h}(k, l)$ and $x^{v}(k, l)$ are robust asymptotically stable in this case.

Let $a_{2}=-2.9$; the stability criteria given in Theorem 3 are feasible by solving LMIs (7), which guarantee the robust asymptotical stability for the underlying uncertain Roessertype discrete-time 2D systems. On the other hand, Figures 3 and 4 show the state trajectory of the system state variables 


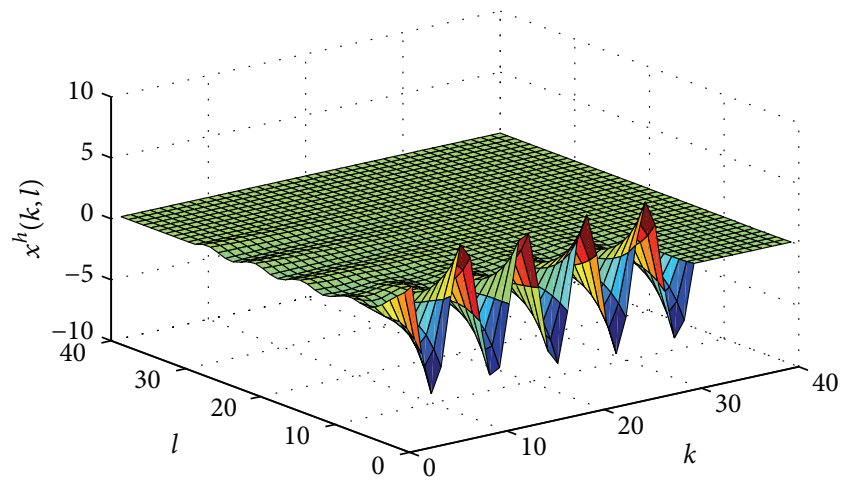

FIgURE 1: The state trajectory of $x^{h}(k, l)$ with $a_{2}=-0.6$.

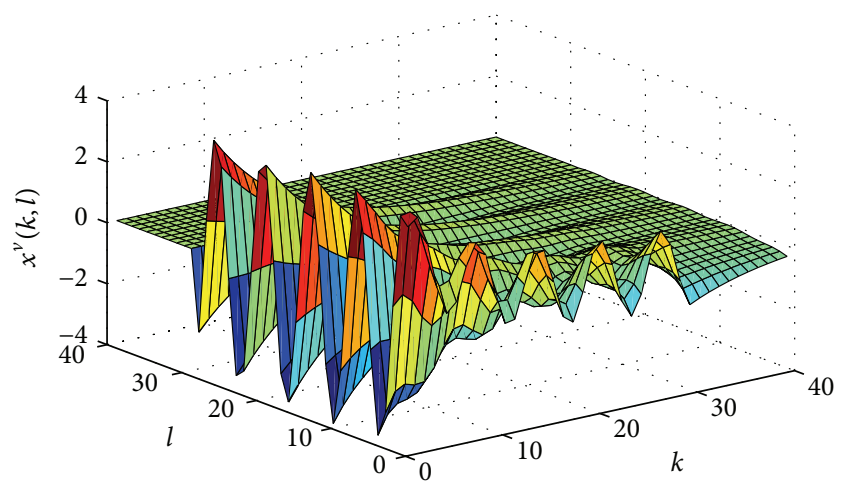

FIgURE 2: The state trajectory of $x^{v}(k, l)$ with $a_{2}=-0.6$.

$x^{h}(k, l)$ and $x^{v}(k, l)$ with $\alpha_{1}=0.4$ and $\alpha_{2}=0.6$, respectively. From Figures 3 and 4 , it is easy to see that the state trajectories of $x^{h}(k, l)$ and $x^{v}(k, l)$ are robust asymptotically stable in this case.

Let $a_{2}=-8.9$; the stability criteria given in Theorem 3 are not feasible by solving LMIs (7), which do not guarantee the robust asymptotical stability for the underlying uncertain Roesser-type discrete-time 2D systems. On the other hand, Figures 5 and 6 show the state trajectory of the system state variables $x^{h}(k, l)$ and $x^{v}(k, l)$ with $\alpha_{1}=0.4$ and $\alpha_{2}=0.6$, respectively. From Figures 5 and 6 , it is easy to see that the state trajectories of $x^{h}(k, l)$ and $x^{v}(k, l)$ are not robust asymptotically stable in this case. Now, it could be concluded that the effectiveness and applicability of the proposed approach given in Theorem 3 are illustrated by means of numerical experiments.

\section{Conclusions}

The problem of robust stability analysis of a class of uncertain Roesser-type discrete-time 2D systems has been addressed by using an efficient parameter-dependent Lyapunov function. In particular, the parameter uncertainties of the underlying 2D system's parameter matrices belong to a convex bounded uncertain domain, which often is named as polytopic uncertainty and appears typically in most practical systems. In order to ensure the robust asymptotic stability of the

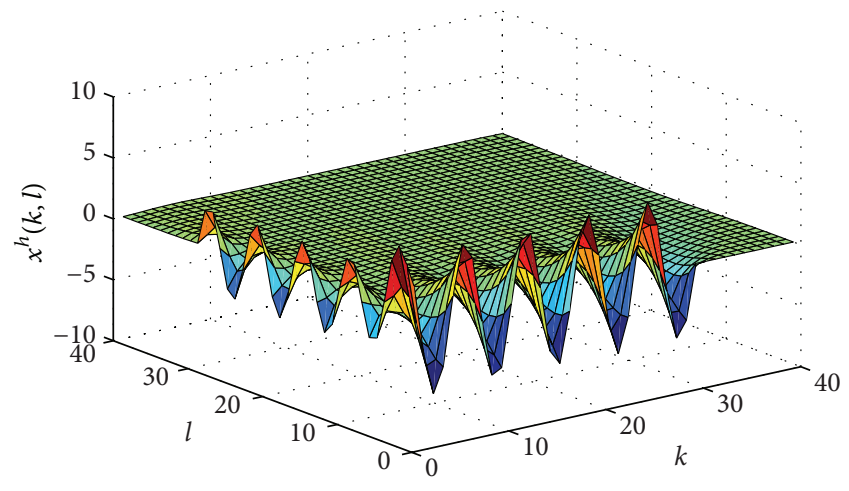

Figure 3: The state trajectory of $x^{h}(k, l)$ with $a_{2}=-2.9$.

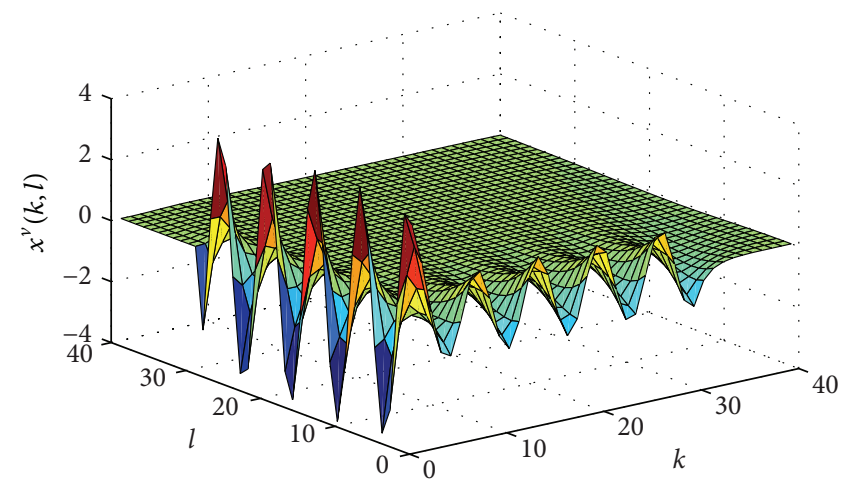

FigURE 4: The state trajectory of $x^{v}(k, l)$ with $a_{2}=-2.9$.

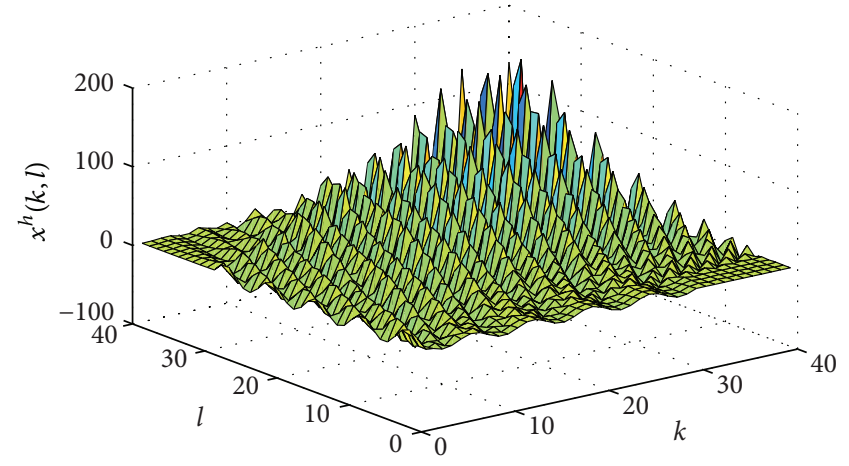

Figure 5: The state trajectory of $x^{h}(k, l)$ with $a_{2}=-8.9$.

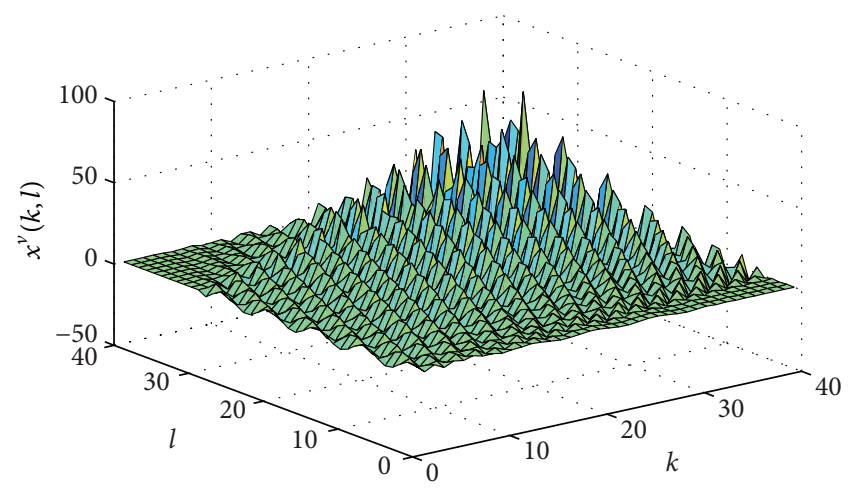

Figure 6: The state trajectory of $x^{v}(k, l)$ with $a_{2}=-8.9$. 
uncertain Roesser-type discrete-time 2D systems, LMI-based robust stability criteria are proposed by exploiting the algebraic properties of the convex bounded uncertain domain. Finally, a numerical example is provided to demonstrate the effectiveness and applicability of the approach given in this paper.

\section{Conflict of Interests}

The authors declare that there is no conflict of interests regarding the publication of this paper.

\section{Acknowledgments}

This work is supported by the National Nature Science Foundation of China under Grant 61304069 and 61372195, the National Nature Science Foundation of Liaoning Province under Grant 2013020124, 2012201010, and 201102160, the Key Project of Chinese Ministry of Education under Grant 212033, the Key Technologies R\&D Program of Liaoning Province under Grant 2011224006, the Program for Liaoning Excellent Talents in University under Grant LJQ2011136, the Scientific Research Fund of Liaoning Provincial Education Department under Grant L2013494, and the Science and Technology Program of Shenyang under Grant F11-264-1-70.

\section{References}

[1] D. Henrion, D. Arzelier, D. Peaucelle, and M. Šebek, "An LMI condition for robust stability of polynomial matrix polytopes," Automatica, vol. 37, no. 3, pp. 461-468, 2001.

[2] S. Yin, H. Luo, and S. Ding, "Real-time implementation of faulttolerant control systems with performance optimization," IEEE Transactions on Industrial Electronics, vol. 64, no. 5, pp. 24022411, 2014.

[3] S. Yin, S. X. Ding, A. H. A. Sari, and H. Hao, "Data-driven monitoring for stochastic systems and its application on batch process," International Journal of Systems Science, vol. 44, no. 7, pp. 1366-1376, 2013.

[4] S. Yin, S. Ding, A. Haghani, H. Hao, and P. Zhang, "A comparison study of basic datadriven fault diagnosis and process monitoring methods on the benchmark Tennessee Eastman process," Journal of Process Control, vol. 22, no. 9, pp. 1567-1581, 2012.

[5] D. Henrion, D. Arzelier, and D. Peaucelle, "Positive polynomial matrices and improved LMI robustness conditions," Automatica, vol. 39, no. 8, pp. 1479-1485, 2003.

[6] P. L. D. Peres and J. C. Geromel, " $\mathscr{H} 2$ control for discrete-time systems optimality and robustness," Automatica, vol. 29, no. 1, pp. 225-228, 1993.

[7] P. L. D. Peres, J. C. Geromel, and J. Bernussou, "Quadratic stabilizability of linear uncertain systems in convex-bounded domains," Automatica, vol. 29, no. 2, pp. 491-493, 1993.

[8] P. Gahinet, P. Apkarian, and M. Chilali, "Affine parameterdependent Lyapunov functions and real parametric uncertainty," IEEE Transactions on Automatic Control, vol. 41, no. 3, pp. 436-442, 1996.

[9] E. Fornasini and G. Marchesini, "State-space realization theory of two-dimensional filters," IEEE Transactions on Automatic Control, vol. 21, no. 4, pp. 484-492, 1976.
[10] R. P. Roesser, "A discrete state-space model for linear image processing," IEEE Transactions on Automatic Control, vol. 20, pp. $1-10,1975$.

[11] D. H. Owens, N. Amann, E. Rogers, and M. French, "Analysis of linear iterative learning control schemes: a 2D systems/ repetitive processes approach," Multidimensional Systems and Signal Processing, vol. 11, no. 1-2, pp. 125-177, 2000.

[12] B. Sulikowski, K. Gałkowski, E. Rogers, and D. H. Owens, "Output feedback control of discrete linear repetitive processes," Automatica, vol. 40, no. 12, pp. 2167-2173, 2004.

[13] B. Sulikowski, K. Galkowski, E. Rogers, and D. H. Owens, "PI control of discrete linear repetitive processes," Automatica, vol. 42, no. 5, pp. 877-880, 2006.

[14] S. Yin, G. Wang, and H. Karimi, "Data-driven design of robust fault detection system for wind turbines, Mechatronics," 2013.

[15] V. Singh, "Elimination of overflow oscillations in 2-D digital filters employing saturation arithmetic: an LMI approach," IEEE Signal Processing Letters, vol. 12, no. 3, pp. 246-249, 2005.

[16] V. Singh, "Stability analysis of 2-D discrete systems described by the Fornasini-Marchesini second model with state saturation," IEEE Transactions on Circuits and Systems II, vol. 55, no. 8, pp. 793-796, 2008.

[17] A. Dhawan and H. Kar, "Optimal guaranteed cost control of 2-D discrete uncertain systems: an LMI approach," Signal Processing, vol. 87, no. 12, pp. 3075-3085, 2007.

[18] V. Singh, "New LMI condition for the nonexistence of overflow oscillations in 2-D state-space digital filters using saturation arithmetic," Digital Signal Processing, vol. 17, no. 1, pp. 345-352, 2007.

[19] V. Singh, "Improved LMI-based criterion for global asymptotic stability of 2-D state-space digital filters described by Roesser model using two's complement arithmetic," Digital Signal Processing, vol. 22, no. 3, pp. 471-475, 2012.

[20] V. Singh, "On global asymptotic stability of 2-D discrete systems with state saturation," Physics Letters A, vol. 372, no. 32, pp. 5287-5289, 2008.

[21] A. Dhawan and H. Kar, "An LMI approach to robust optimal guaranteed cost control of 2-D discrete systems described by the Roesser model," Signal Processing, vol. 90, no. 9, pp. 2648-2654, 2010.

[22] A. Dey and H. Kar, "Robust stability of 2-D discrete systems employing generalized overflow nonlinearities: an LMI approach," Digital Signal Processing, vol. 21, no. 2, pp. 262-269, 2011.

[23] A. Dhawan and H. Kar, "An improved LMI-based criterion for the design of optimal guaranteed cost controller for 2-D discrete uncertain systems," Signal Processing, vol. 91, no. 4, pp. 10321035, 2011.

[24] V. Singh, "New approach to stability of 2-D discrete systems with state saturation," Signal Processing, vol. 92, no. 1, pp. 240247, 2012.

[25] H. Kar, "A new criterion for the global asymptotic stability of 2-D state-space digital filters with twos complement overflow arithmetic," Signal Processing, vol. 92, no. 9, pp. 2322-2326, 2012.

[26] A. Dey and H. Kar, "An LMI based criterion for the global asymptotic stability of 2-D discrete state-delayed systems with saturation nonlinearities," Digital Signal Processing, vol. 22, no. 4, pp. 633-639, 2012.

[27] W. Sun, H. Gao Sr., and O. Kaynak, "Finite frequency $\mathscr{H}_{\infty}$ control for vehicle active suspension systems," IEEE Transactions on Control Systems Technology, vol. 19, no. 2, pp. 416-422, 2011. 
[28] W. Sun, Y. Zhao, J. Li, L. Zhang, and H. Gao, "Active suspension control with frequency band constraints and actuator input delay," IEEE Transactions on Industrial Electronics, vol. 59, no. 1, pp. 530-537, 2012.

[29] Y. Suzuki, S. Morioka, and H. Ueda, "Cooking support with information projection over ingredient," International Journal of Innovative Computing, Information and Control, vol. 9, no. 12, pp. 4753-4763, 2013.

[30] M. Nakatani and T. Ohno, "An integrated model depicting psychology of active/Non-active internet users: how to motivate people to use internet at home," International Journal of Innovative Computing, Information and Control, vol. 9, no. 12, pp. 4765-4779, 2013. 


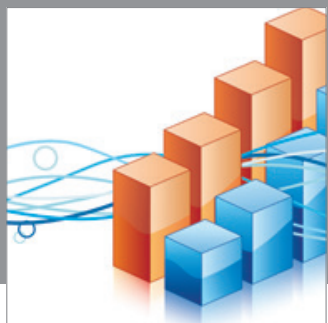

Advances in

Operations Research

mansans

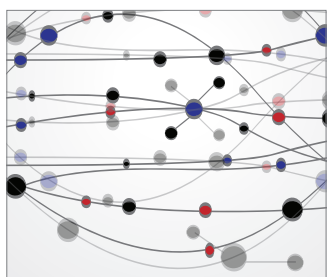

The Scientific World Journal
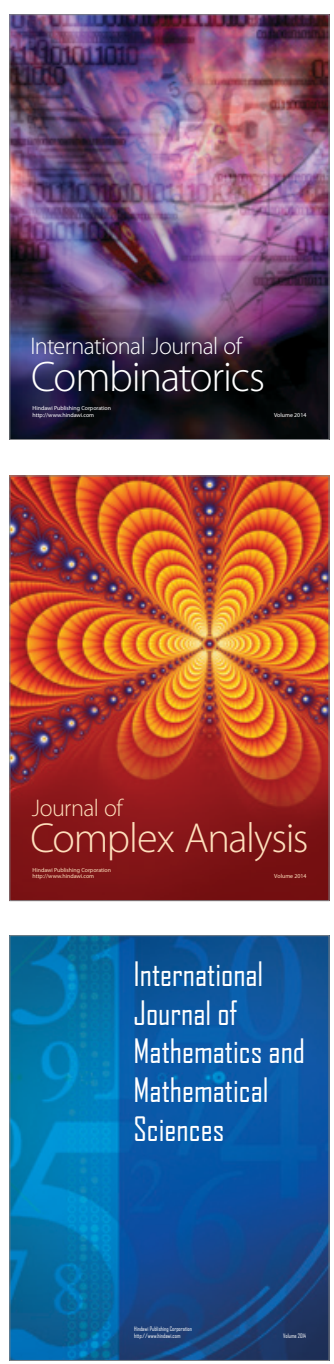
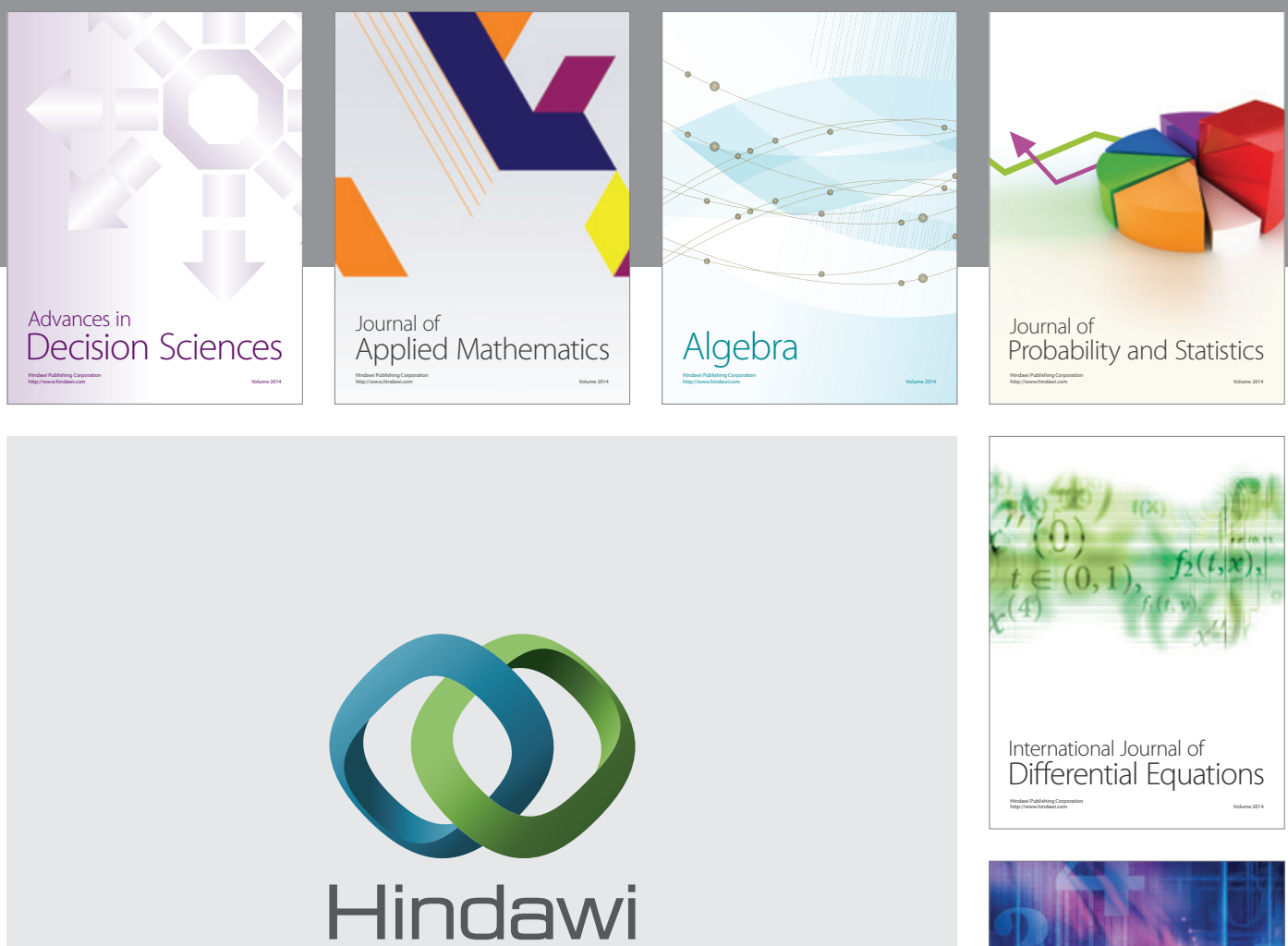

Submit your manuscripts at http://www.hindawi.com
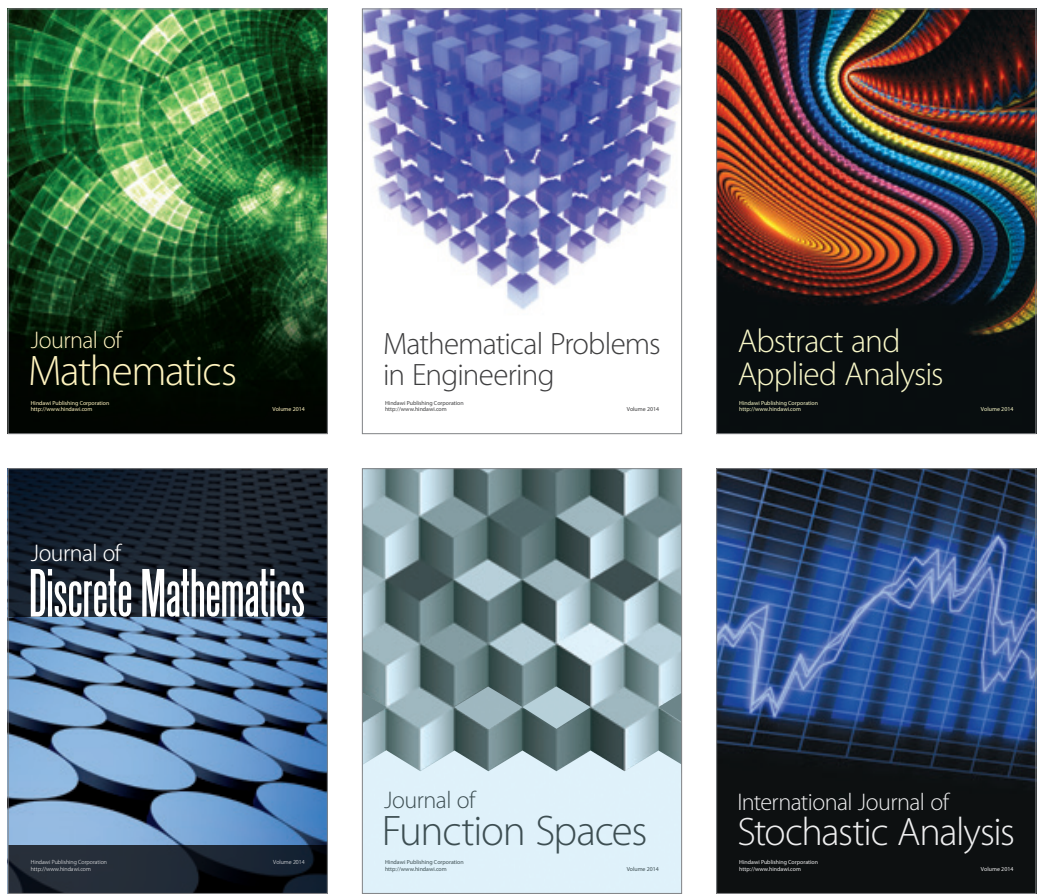

Journal of

Function Spaces

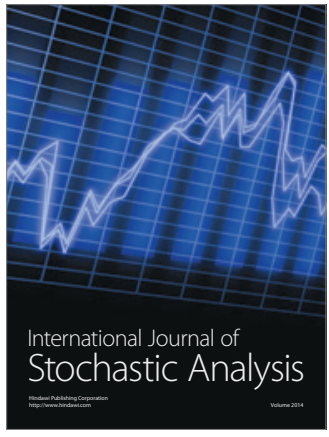

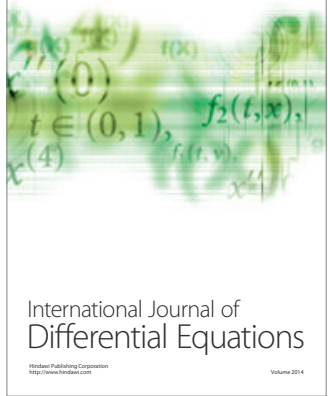
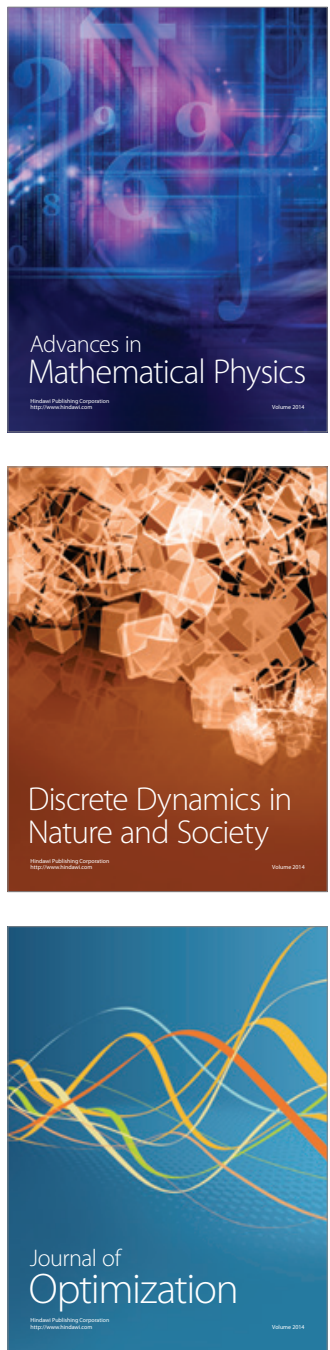